\title{
Recombinant protein of Haemonchus contortus small GTPase ADP-ribosylation factor 1 (HCARF1) modulate the cell mediated immune response in vitro
}

\author{
Javaid Ali Gadahi ${ }^{1,2}$, Muhammad Ehsan ${ }^{1}$, Shuai Wang ${ }^{1}$, Zhenchao Zhang ${ }^{1}$, Ruofeng \\ Yan ${ }^{1}$, Xiaokai Song ${ }^{1}$, Lixin $\mathbf{X u}^{1}$ and Xiangrui $\mathbf{L i}^{1}$ \\ ${ }^{1}$ College of Veterinary Medicine, Nanjing Agricultural University, Nanjing, PR China \\ 2 Department of Veterinary Parasitology, Sindh Agriculture University, Tando Jam, Pakistan \\ Correspondence to: Xiangrui Li, email: lixiangrui@njau.edu.cn \\ Keywords: H. contortus; ADP-ribosylation factor; PBMCs; cytokines; proliferation \\ Received: February 23, $2017 \quad$ Accepted: November 03, $2017 \quad$ Published: November 26, 2017
}

Copyright: Gadahi et al. This is an open-access article distributed under the terms of the Creative Commons Attribution License 3.0 (CC BY 3.0), which permits unrestricted use, distribution, and reproduction in any medium, provided the original author and source are credited.

\section{ABSTRACT}

ADP-ribosylation factors (ARFs) are members of the Ras-related small GTPase family involved in the vesicular trafficking regulation. Immunomodulatory effects of these proteins on host cell are not being addressed yet. $H$. contortus small GTPase ADP-ribosylation 1 gene (HCARF1) was cloned and recombinant protein of HCARF1 (rHCARF1) was successfully expressed in Escherichia coli. Binding activity of rHCARF1 to goat PBMCs was confirmed by immunofluorescence assay (IFA) and its immunomudulatory effects on cytokine secretion, cell proliferation, cell migration and nitric oxide production (NO) were observed by co-incubation of rHcARF1. IFA results revealed that rHCARF1 could bind to the PBMCs. The interaction of rHCARF1 modulated the cytokine production, the production of IL-4, IL-10 and IL-17 was increased in a dose dependent manner, however, the IFN-Y production was significantly decreased. Cell migration and NO production were significantly increased by rHCARF1, whereas, rHcARF1 treatment significantly suppressed the proliferation of the PBMC in a dose dependent manner. Our findings showed that the rHcARF1 play important roles on the goat PBMCs.

\section{INTRODUCTION}

ADP-ribosylation factors (ARFs) are member of the Ras-related small GTPases family also known as low molecular weight guanine-nucleotide-binding $(\mathrm{G})$ proteins [1] and their involvement in the vesicular trafficking regulation has been well characterized [2]. ARF1 is characteristically related with the golgi and in some cell types also be found related with the plasma membrane and is an important regulator of the biological process induced by epidermal growth factor [3-7]. Involvement of the ARF1 in the activation of signaling molecules, such as phospholipase D, PI3K and type I phosphatidylinositol 4-phosphate 5-kinase has been reported, these data suggests that in addition of trafficking regulation, ARF1 GTPase also act as a signal transducer [5, 8-10].

ARF1 is involved in membrane affinity and it actively involve in the formation of non-clatherin/clatherin coated vesicles which helps in the transportation of vesicles to carry important cellular components required for biological processes such as cell signaling [11]. ARF1 also involved in the activation of PLD enzyme which cleaves phophatidylcholine to generate phophatidic acid (PA) and choline. PA could modulate many cellular events like DNA synthesis, cell proliferation, and secretory responses [12]. Characterization of the ARF proteins has been performed in various parasites included Caenorhabditis elegans [13, 14], Entamoeba histolytica [15], Plasmodium falciparum [16, 17] and Leishmania [18].

Haemonchus contortus is an abomasal nematode parasite, it is the most important parasitic problem on a global basis [19]. H. contortus is responsible for the decline of rural economy due to weight loss and anemia resulted in decreased meat and milk production and $H$. contortus is one of the comprehensively used parasitic 
nematode for drug discovery, vaccine development and drug resistance [20-23]. The animal's body triggers several defense mechanisms during $H$. contortus infection to control the infection in different ways, such as reactive oxygen species production by immune cells. This protection may leads to damages of various host cells and tissues by oxidative stress [24, 25].

Previously, we identified that ARF1 protein was one of the interacting protein with goat PBMCs at multiple developmental stages in vivo [26]. Molecular cloning and functional characterization of $H$. contortus ARF1 has not being addressed yet. In the current study, recombinant protein of HcARF1 (rHcARF1) was constructed and its immunomodulatory effects on the goat PBMCs was evaluated.

\section{RESULTS}

\section{Sequence and phylogenetic analysis}

The recombinant plasmid pET32a-HcARF1 was confirmed by restriction enzyme digestion and sequencing. The results of the BLASTx revealed that, ORF contains $546 \mathrm{bp}$ encodes 181 amino acids. The deduced protein sequence of HcARF1 was used for multiple sequence alignment (Figure 1A). The results of the multiple alignments showed that HcARF1 is very close to the ADP-ribosylation factor family protein of Ancylostoma ceylanicum (98\%), Dictyocaulus viviparous (98\%), Necator americanus (98\%), Loa loa (97\%), Strongyloides ratti (97\%), Wuchereria bancrofti (97\%), Pristionchus pacificus (97\%) Caenorhabditis elegans (96\%), The typical characteristics of the HcARF1 were confirmed as ARF $1-5$ by their GTP/Mg2 binding and putative GAP interaction sites (Figure 1B). These findings confirmed that, the cloned ORF belongs to the $H$. contortus ARF1 family. The phylogenic tree analysis indicated that HcARF 1 was closely related to ARF of homologous protein sequence obtained from NCBI data base (Figure $1 \mathrm{C})$.

\section{Expression and purification of rHcARF1}

The recombinant protein of HcARF1 was expressed and purified as a His tagged fusion protein. The expressed protein was detected at $38 \mathrm{kDa}$, it is higher than the calculated mass of $20 \mathrm{kDa}$ of HcARF1 because of extra molecular mass of pET 32a expression vector (Figure 2).

\section{Detection of recombinant HcARF1 protein by immunoblotting}

The results of the immunoblot indicated that the rHcARF1 was detected by rat anti rHcARF1, but in negative control no protein was identified by normal rat sera (Figure 3).

\section{Interaction of rHcARF1 with goat PBMCs}

Immunofluorescence assay (IFA) was used to confirm the interaction of rHcARF1 with host PBMCs.

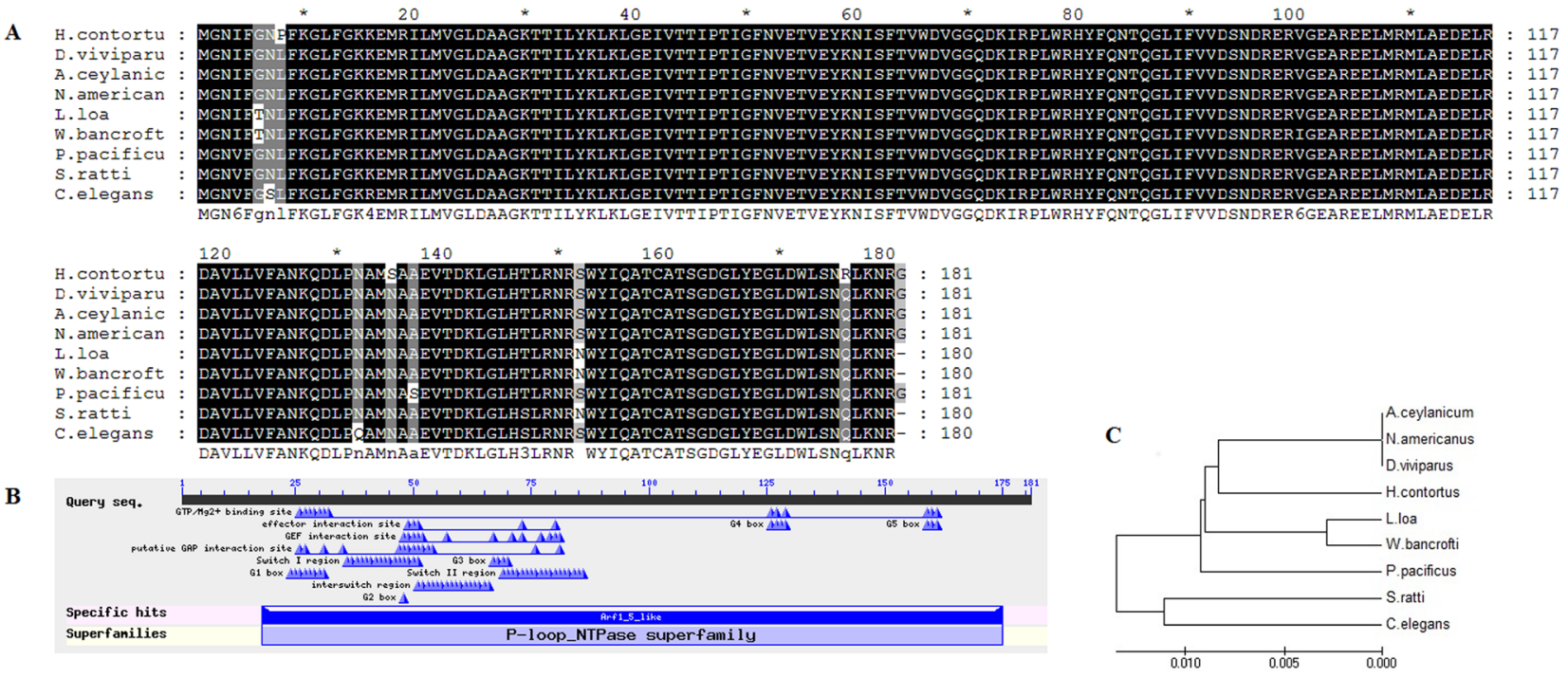

Figure 1: Multiple alignment of amino acid sequence of HcARF1. A. The amino acid sequence of HcARF1 aligned with other organism ARF family reported in the NCBI database of Ancylostoma ceylanicum (98\%), Dictyocaulus viviparous (98\%), Necator americanus (98\%), Loa loa (97\%), Strongyloides ratti (97\%), Wuchereria bancrofti (97\%), Pristionchus pacificus (97\%), Caenorhabditis elegans $(96 \%)$. B. Putative conserved domain. C. Phylogenetic analysis of the relationships among the amino acid sequences of HcARF1 and known similar sequences by minimum evolution. 
Confocal microscopy indicated that rHcARF1 was interacted with the cell surface (red fluorescence). In the control group, no binding was observed (Figure 4).

The binding of rHcARF1 to goat PMBCs increased IL-4, IL-10 and IL-17 and suppressed IFN- $\gamma$

In the present study ELISA was used to analyze the impacts of the rHcARF1 on the cytokine production. Our findings indicated that rHcARF1 modulating the cytokine production (Figure 5). Secretion of IL-4, IL-10 and IL-17 was significantly increased whereas, the production of the IFN- $\gamma$ was decreased in PBMCs incubated with different concentration of rHcARF1.

Interaction of rHcARF1 with goat PBMCs significantly increased the production of cytokine IL-4, IL10 and IL-17 in a dose dependent manner. On the contrary, type II interferon (IFN- $\gamma$ ) was decreased by the interaction of rHcARF1 (Figure 5).

\section{The interaction of rHcARF1 with goat PMBCs increased cell migration}

In the current study, cell migration assay was performed to appraise the effect of rHcARF1 on cell

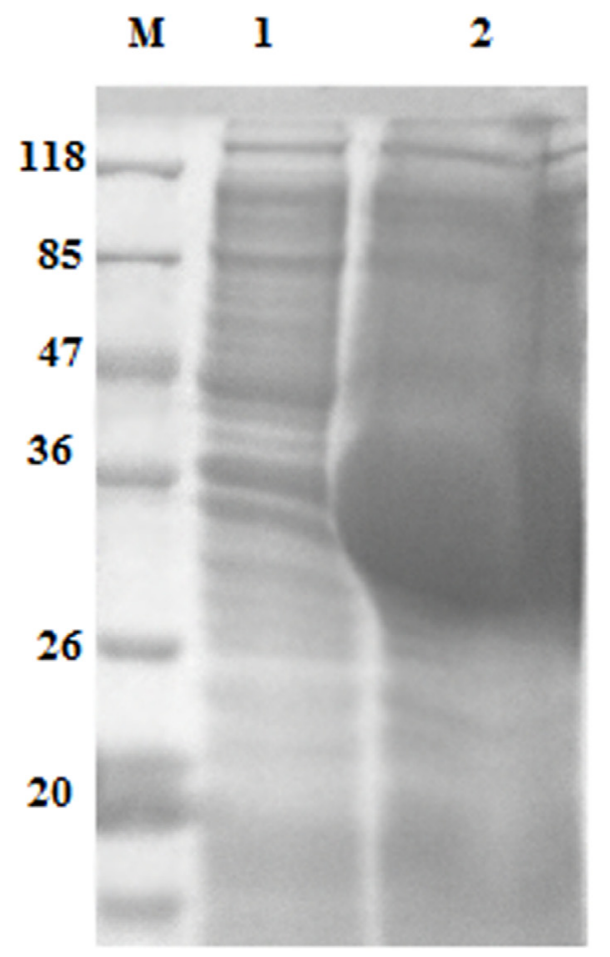

Figure 2: Expression of rHcARF1 protein after induction with $1 \mathrm{mM}$ IPTG. Lane M: standard protein molecular weight marker, 1: recombinant expression vector before induction, Lane 2 expression after induction. migration (Figure 6). Our findings showed that cell migration was significantly increased in cells incubated with 20 and $40 \mu \mathrm{g} / \mathrm{ml}$ of rHcARF1.

\section{The interaction of rHcARF1 with goat PMBCs decreased cell proliferation}

The treatment of rHcARF1 significantly decreased the multiplication of the PBMC at the concentration $40 \mu \mathrm{g} /$ $\mathrm{ml}$ as compared to the control group (Figure 7).

\section{The binding of rHcARF1 to goat PMBCs increased nitric oxide production}

Total nitric oxide assay kit was used to measure the Nitric oxide (NO) production by PBMCs incubated with various concentration of rHcARF1. Our findings showed that, $\mathrm{rHcARF} 1$ significantly increased the $\mathrm{NO}$ production at 10,20 and $40 \mu \mathrm{g} / \mathrm{ml}$ (Figure 8 ).

\section{DISCUSSION}

A GTP-binding protein has been concerned in the regulation of membrane traffic by the secretory pathway and ARFs are highly conserved family of eukaryotic small GTP-binding proteins with roles in membrane dynamics

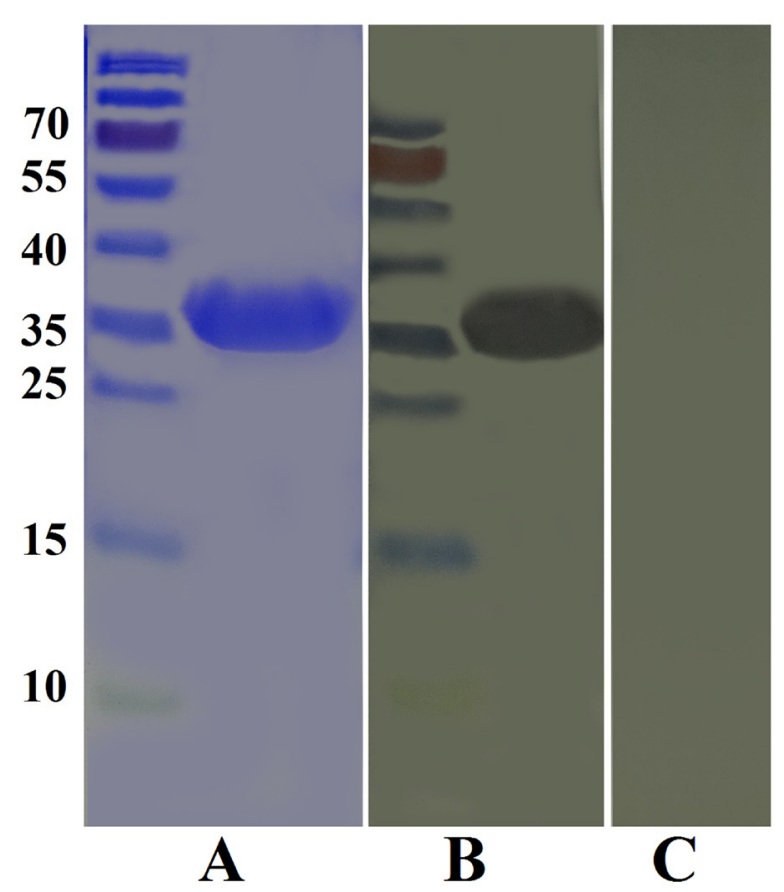

Figure 3: Western blot analysis of rHcARF1. A. Purified rHcARF1 was electrophoresed in SDS-PAGE and stained with Coomassie blue, B. then transferred to a membrane for western blot analysis with rat anti-rHcARF1 sera and $\mathbf{C}$. normal rat sera as control. 


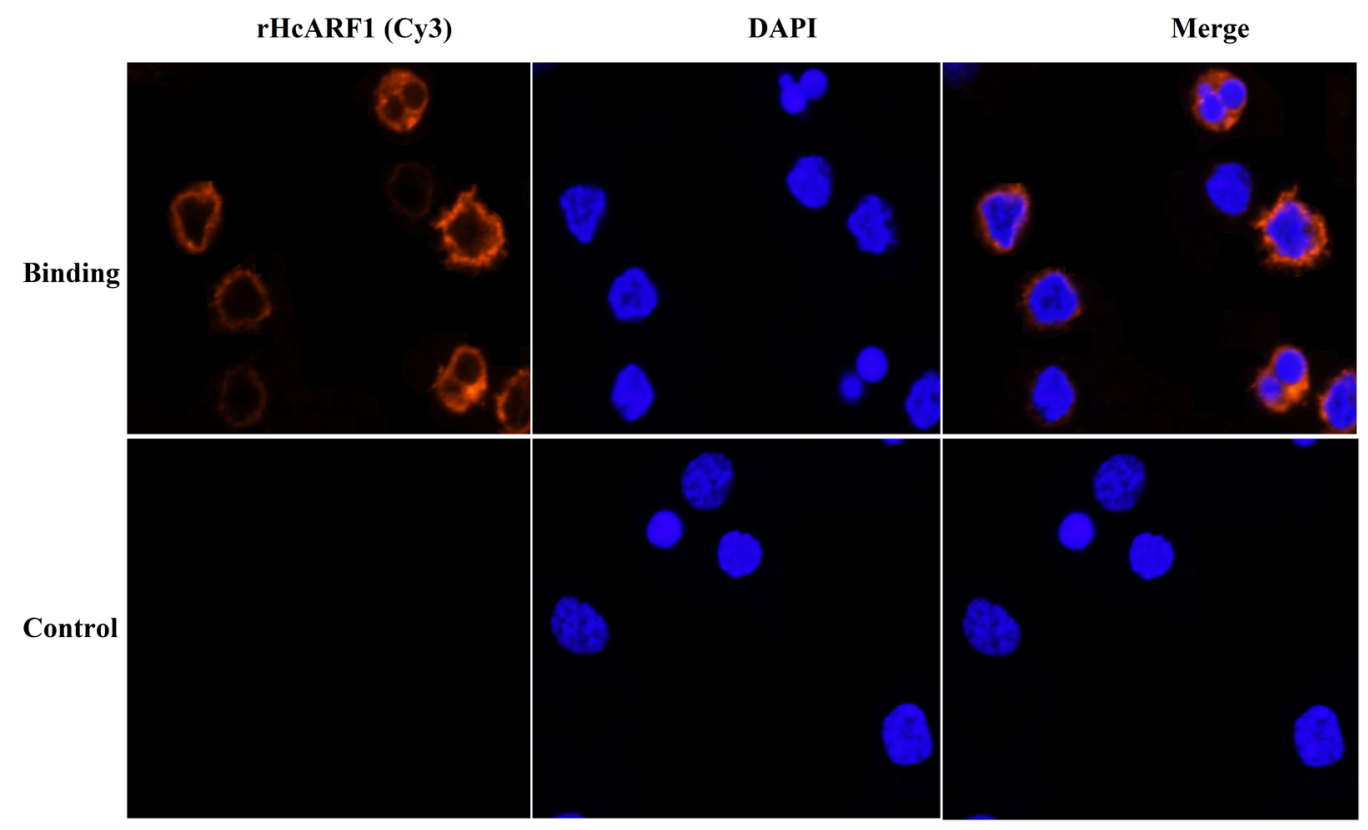

Figure 4: Confirmation of binding of rHcARF1 to goat PBMCs by IFA. The nuclei of the corresponding cells were visualized by DAPI (blue) staining. Staining of the target proteins (red) were visualized by Cy3-conjugated secondary antibody. Merge, overlap of red and blue channels. No red fluorescence was observed in control group.
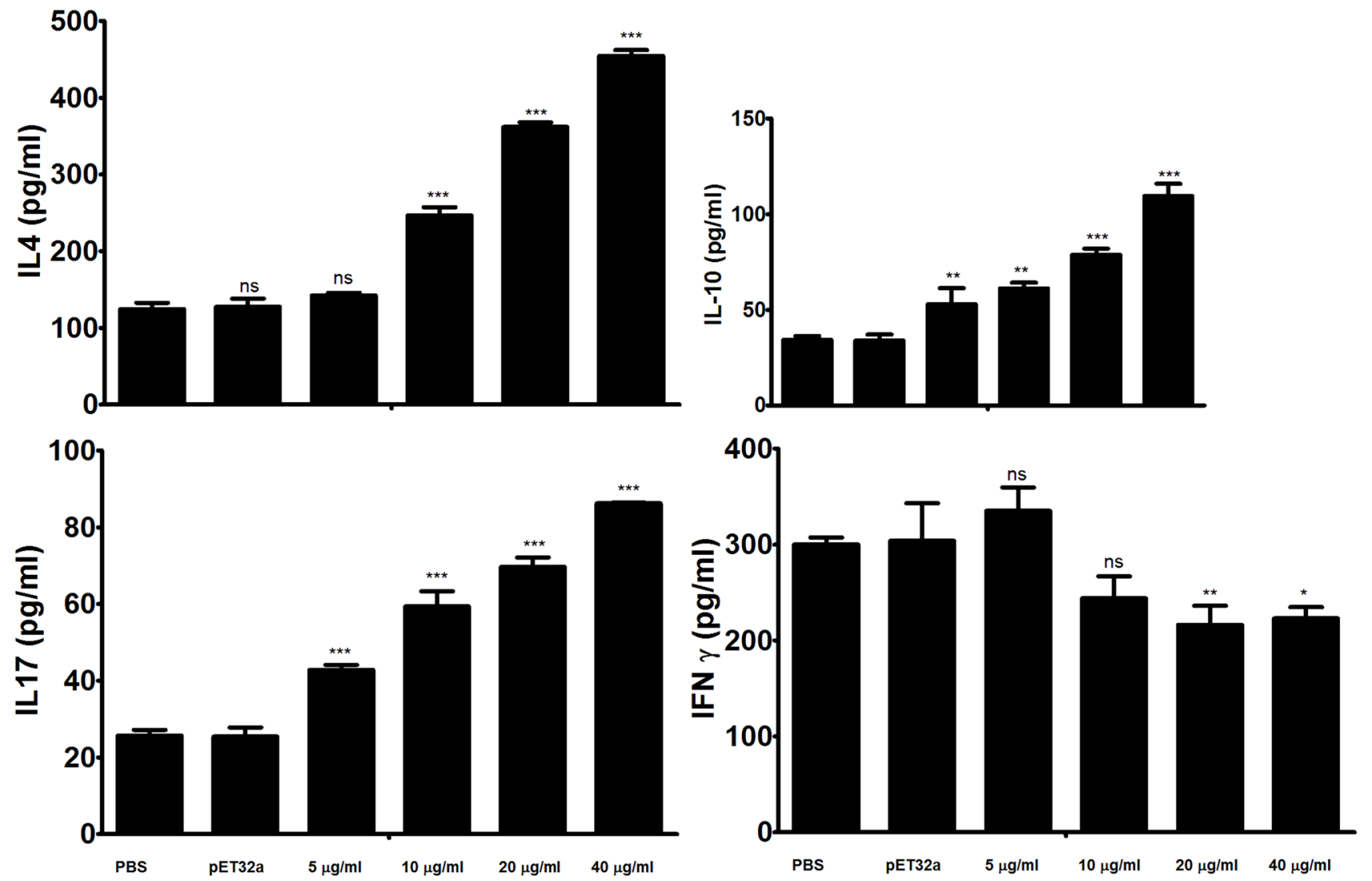

Figure 5: Analysis of the level of multiple cytokine production by PBMCs in vitro. PBMCs were stimulated with ConA (10 $\mu \mathrm{g} / \mathrm{ml}$ ) for $24 \mathrm{~h}$ in the presence or absence of various concentrations of rHcARF1 and pET32a. Cytokine secretion in the supernatant of cell cultures was quantified by ELISA. The data are representative of three independent experiments $\left({ }^{*} p<0.01,{ }^{* *} p<0.001\right.$, ns non significant). 


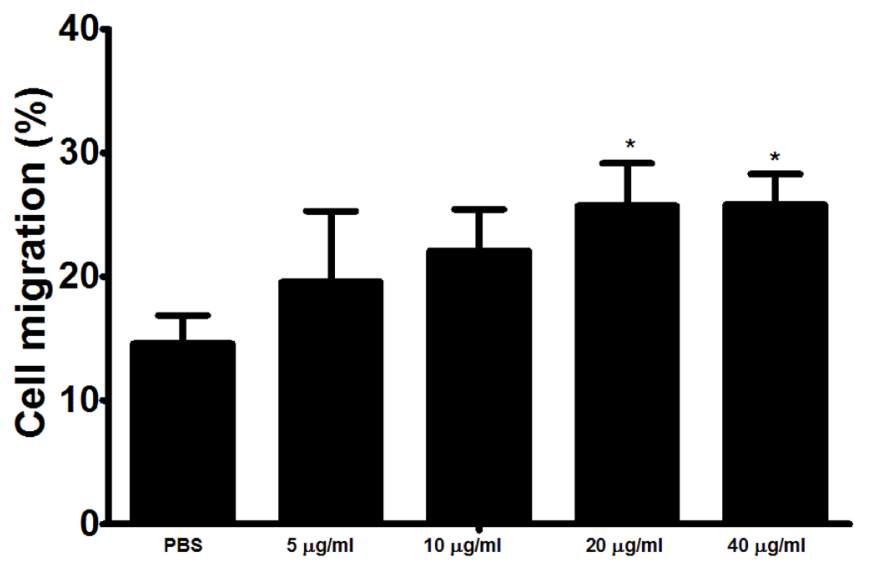

Figure 6: Impact of the various concentration of rHcARF1 on PBMC migration. PBMC were treated with control buffer and different concentrations of rHcARF1, Then the random migration was determined. The difference between the mean values was calculated using ANOVA. Data are representative of 3 independent experiments; ${ }^{*} p<0.01$ versus the control.

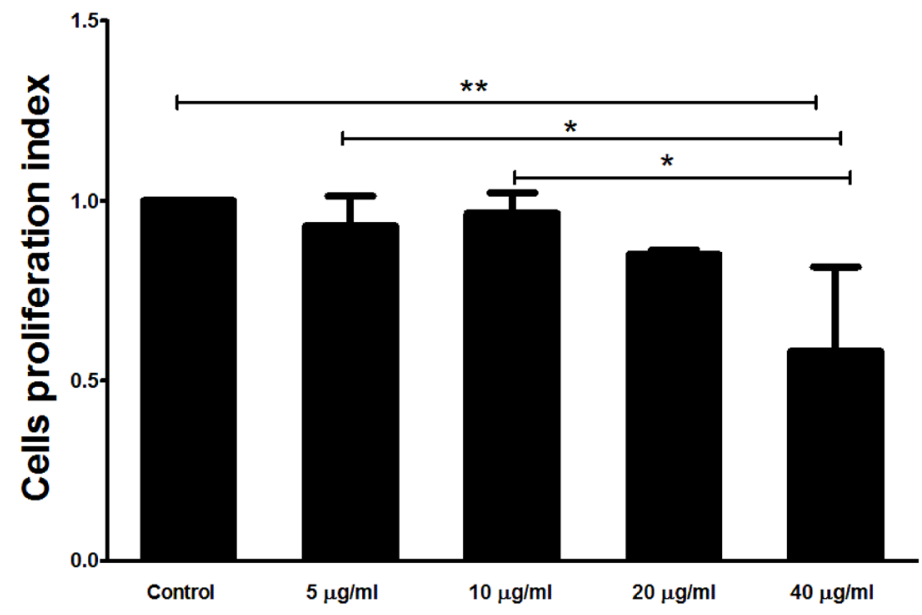

Figure 7: Effects of rHcARF1 on PBMCs proliferation. Cells were activated with ConA and incubated at the same time with serial concentrations of $\mathrm{rHcARF} 1$ at $37^{\circ} \mathrm{C}$ and $5 \% \mathrm{CO}_{2}$. The proliferation was measured by CCK-8 incorporation after $72 \mathrm{~h}$. Cell proliferation index was calculated considering the $\mathrm{OD}_{450}$ values in controls as $100 \%$. The data were representative of three independent experiments $\left({ }^{*} p<0.01\right.$ and $\left.{ }^{* *} p<0.001\right)$.

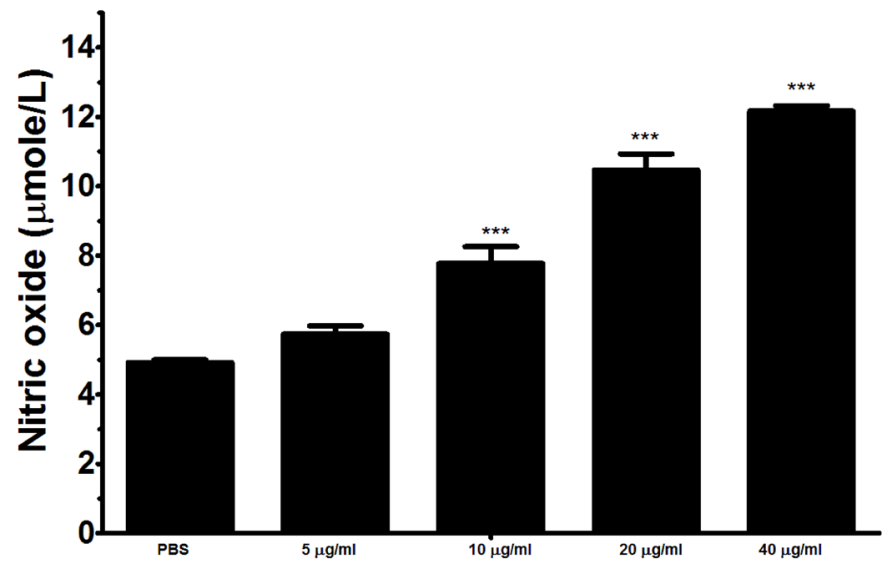

Figure 8: Effects of rHARF1 on nitric oxide production by PBMCs in vitro. Cells were activated with ConA and incubated at the same time with serial concentrations of rHcARF 1 at $37^{\circ} \mathrm{C}$ and $5 \% \mathrm{CO}_{2}$. The nitrite concentration in the PBMCs was measured by using the Griess assay and used as an indicator of nitric oxide production by the PBMCs. The data were representative of three independent experiments $\left({ }^{* * *} p<0.0001\right)$. 
and vesicle trafficking $[27,28]$. ARFs proteins also being identified in different parasites [13, 15, 29-32].

To date, little information is available on the ARFs in the parasitic nematode, in case of $H$. contortus molecular and functional characterization has not been done. In the current research we have cloned a small GTPase ARF1 gene from the Haemonchus contortus cDNA. The multiple sequence alignment of HcARF1 with other organism indicated that the phylogenetic relationship of $H$. contortus was closely related with the nematodes (Ancylostoma ceylanicum, Angiostrongylus cantonensis, Dictyocaulus viviparous, Dirofilaria immitis and Loa loa). The multiple sequence alignment of HcARF1 indicated that cloned gene belongs to the ARF protein family.

Cytokine produced by the immune cells are responsible for communication as well as regulation of the immune system. First line of the defense against various organisms including parasites provided by the innate immune system by toll-like receptors (TLRs) family of pattern recognition receptors (PRRs) that recognize the broad range of pathogen-associated molecular patterns (PAMPs) $[33,34]$.

In the present study, rHcARF1 increased cytokines IL-4, IL-10 and IL-17 secretion by PBMCs but decreased the secretion of Type II interferon (IFN- $\gamma$ ). Previously, it was suggested that an ADP ribosylation factor-GTPase activating protein 1 was negatively regulated LPSinduced pro-inflammatory mediators production by downregulation of LPS signaling [35]. Wu et. al reported that, class III PI3K/ARF6-dependent pathway was involved in regulating cellular subsequently modulates CpGODN/ TLR9-signaling cascades such as TLR9 trafficking and cytokine production [36]. In our study, cytokine secretion modulated by rHcARF1 strongly indicated the multiple and distinct regulatory effects of ARF on goat immune cells.

It is generally considered that, type 2 immunity (Th2) associated with secretion of IL-4 and IL-5 is the main immune mechanism against helminths including H. contortus [37-39]. In our previous study we described that interaction of HcESPs with goat PBMCs decreased the production of IL-4 in vitro [40]. The findings of our study conflicting the above results. Here, we found that the rHcARF1 could increase the production of IL-4. These results directed that $\mathrm{rHcARF} 1$ might be played a part in the initiation of the Th2 immunity. However, HcESPs are the group of various proteins, thus proteins involved in decline of IL-4 are worthy of further investigations.

IL-17 a cytokine is strong inducer of inflammation produced by Th17 cells [41] and it is concerned with pathogenesis [42-48]. In our previous studies we found that HcARF1 could interact with host PBMCs at various developmental stages of $H$. contortus [26] and the secretion of IL-17 was increased by the interaction of HcESPs [40]. Here, we reported that rHcARF1 enhanced the production of IL-17, which indicated that rHcARF1 could part play some roles in the HcESPs on IL-17 production.

It has been recognized that immune suppressive cytokine IL-10 secreted by inducible Treg cells ( $\mathrm{iT}_{\mathrm{Reg}}$ ) suppress the IFN- $\gamma$ production $[49,50]$. The generation of immune suppressive cytokine IL-10 might be an important tactic by which parasites could suppress the IFN- $\gamma$-dependent, cell-mediated immunity $[51,52]$. In our previous research we found that rHcESPs suppressed the immune response by increasing the IL-10 and decreasing IFN- $\gamma$ production [40]. Here we found that interaction of rHcARF1 with goat PBMCs could increase the IL-10 and decrease the IFN- $\gamma$ production. Therefore, we suggested that, $\mathrm{rHcARF} 1$ is very important protein of HcESPs that could suppress the Th1 immune response and might be beneficial for $H$. contortus evading from the host immunity at early stage.

Previous studies demonstrated that ARF could be regulating the cell cycle progression by the suppression of transcription factor E2F1 activity [53, 54]. Currently, the effects of the rHcARF1 on the cell proliferation was evaluated and results indicated that $\mathrm{rHcARF} 1$ significantly inhibited the cell proliferation. Complex regulatory activities was ultimately linked with each other, such as cell activation, cytokine secretion and cell cycling leading to cell proliferation [55].

NO has been reported as an important immunemediator and play important role in immunoregulation in various infections including $H$. contortus by mediating host protection by parasite killing or by suppressing the growth [56-58]. Previously reported that, endogenous IL17 was involved in T cell-mediated NO production [59, $60]$. In the present study immune-modulating effects of the rHcARF1 on the NO production by goat PBMCs was evaluated. Cells incubated with rHcARF1 significantly increased the NO production in a dose dependent manner. This up-regulation might be associated with the increased level of IL-17. Our results indicated that, up-regulation of IL-17 as well as NO production might involve in the TH17/NO inflammatory response and pathogenesis.

In Conclusion, we firstly cloned the HcARF1 and demonstrated that HcARF1 is one of the active protein of HcESPs that might be involved in the immune modulation. These findings indicated that, the interaction of rHcARF1 with host cells increased the production of IL-4, IL-10, IL-17, NO and cell migration. However, the secretion of IFN- $\gamma$ and proliferation of PBMCs was significantly decreased. Our results might help to understand the mechanism involved in host parasite interaction. The immune system has different cell populations including $\mathrm{T}$ and $\mathrm{B}$ lymphocytes, macrophages, antigen presenting cells, NK cells, etc. How does HcARF1 activate immune and cellular response and which immune cells have been actively involved during the infection need to be further researched. 


\section{MATERIALS AND METHODS}

\section{Ethics statement}

Animal experiments were conducted following the guidelines of the Animal Ethics Committee, Nanjing Agricultural University, China. All experimental protocols were approved by the Science and Technology Agency of Jiangsu Province. The approval ID is SYXK (SU) 20100005 .

\section{Synthesis of Haemonchus contortus cDNA}

Total RNA was isolated from adult worms of $H$. contortus collected from the abomasums of donor goats as described previously [61]. The worms were ground using a pre-chilled mortor and pestle. One $\mathrm{ml}$ of Trizol (Invitrogen) was added and homogenized for 30 minutes. Then $200 \mu 1$ of Tri-chloromethane was added and the mixture was spun at $12,000 \mathrm{rpm}$ for $15 \mathrm{~min}$ at $4^{\circ} \mathrm{C}$. After that, RNA was precipitated from the supernatant by the addition of 0.25 volumes of isopropyl alcohol per each milliliter of Trizol and incubated at $-20^{\circ} \mathrm{C}$ for $30 \mathrm{~min}$. The RNA was pelleted at $12,000 \mathrm{rpm}$ at $4^{\circ} \mathrm{C}$ for $10 \mathrm{~min}$. Hereafter, RNA pellets were washed by $70 \%$ ethanol and dried. The Pellets were resuspended in DEPC-treated water and the RNA solution was used in subsequent cDNA preparation immediately. The cDNA was synthesized by reverse transcription reaction using cDNA Kit (TaKaRa Biotechnology) according to the manufacturer's instructions.

\section{Molecular cloning of HcARF1 and expression of recombinant HcARF1 protein (rHcARF1)}

The complete open reading frame (ORF) of HcARF1 was amplified by reverse transcriptionpolymerase chain reaction (RT-PCR) using the designed primers of $H$. contortus ARF gene (GI: 533372025), gene bank accession number HF964523.1. The sense and antisense primer sequences are as the following: 5'- GGATCCATGGGTAACATTTTCGG -3' and 5'CTCGAGTTATCCTCTGTTTTTCA -3.

The PCR products were purified by using E.Z.N.A. Gel Extraction Kit (Omega bio-tech, USA) and ligated into pMD19-T cloning vector (TaKaRa Biotechnology, China) and then transformed into $E$. coli $\mathrm{DH}_{5 \alpha}$ strain. The positive clones were confirmed by double digestion with BamHI/ Xhol enzymes, and the selected positive recombinant clones were sequenced by Invitrogen Bio-tech (Shanghai, China). The sequence data was assembled and analyzed by DNAssist software version 2.2. The HcARF1 gene was then cloned into BamHI/Xhol sites of expression plasmid
pET32a (+) vector (Novagen, USA). The recombinant plasmid was sequenced to confirm the correct insertion of HcARF1 gene in the proper reading frame.

The expression of the recombinant fusion protein in E. coli BL-2 1 cells (DE3) was induced by isopropyB D -thiogala ctoside (IPTG ) at a final concentration of $1 \mathrm{mM}$ for $4 \mathrm{~h}$ at $37^{\circ} \mathrm{C}$ in Luria-Bertini (LB) medium with ampicillin $(100 \mu \mathrm{g} / \mathrm{ml})$. The histidine-tagged fusion protein was purified from the supernatant of bacterial lysates using the His $\bullet$ Bind ${ }^{\circledR}$ Resin Chromatography kit (Novagen) and dialyzed in phosphate buffered saline (PBS, pH 7.4) to remove imidazole. Endotoxins were removed from the recombinant proteins using ToxinEraser ${ }^{\mathrm{TM}}$ Endotoxin Removal kit (GeneScript, USA). The purity and concentration of the purified rHcARF1 was analyzed by $12 \%$ sodium dodecyl sulfate polyacrylamide gelelectrophoresis (SDS- PAGE) followed by Coomassie blue staining.

\section{Sequence alignments and phylogenetic analysis of HcARF1}

Sequence similarity was assessed using proteinprotein basic local alignment search tools BLASTp and BLASTx sequences (http://www.blast.ncbi.nlm.nih. gov/Blast.cgi). HcARF1 sequences were aligned using ClustalX 1.83 program (http://www.clustal.org/). The phylogenetic tree was constructed by aligning the amino acid sequences using the Neighbor-Joining method and plotted and visualized using the Molecular Evolutionary Genetics Analysis 5.1 program (http://www.megasoftware. net/).

\section{Generation of polyclonal antibodies}

To generate polyclonal antibodies against rHcARF1 , $0.4 \mathrm{mg}$ of rHcARF1 was mixed with Freund's complete adjuvant (1:1) and injected subcutaneously into 3 female Sprague Dawley (SD) rats [62, 63]. Rats received four doses of injection with the same proteins at 2-week intervals. Ten days after the last injection, the rats were anesthetized with diethyl ether, and sera containing specific anti-rHcARF1 antibodies were collected. The concentration of antibodies was determined by ELISA. The specific reactivity with $\mathrm{rHcARF} 1$ was confirmed by western blot analysis.

\section{Immuno-blot for the rHcARF1}

Purified rHcARF1 were resolved by $10 \%$ SDSPAGE and transferred to polyvinylidene difluoride (PVDF) Membrane (Millipore, USA). Non-specific binding was blocked by incubating the membranes in 5\% skim milk in Tris-buffered saline containing $0.1 \%$ Tween-20 (TBST) 
for $1 \mathrm{~h}$ at room temperature. The membranes were then washed 5 times ( 5 min each) with TBST, followed by incubation with the primary antibodies (anti-rHcARF1) for $1 \mathrm{~h}$ at $37^{\circ} \mathrm{C}(1: 100$ dilution in TBST). After washing 5 times with TBST, the membranes were incubated with HRP-conjugated rabbit anti-rat IgG (Sigma, USA) for $1 \mathrm{~h}$ at $37^{\circ} \mathrm{C}$ (diluted 1:2000 in TBST). Finally, the bound antibody was detected using 3,3-diaminobenzidine tetra hydrochloride (DAB) kit (Boster Bio-technology) according to manufacturer's instructions.

\section{Binding of rHcARF1 to goat PBMC}

Freshly isolated PBMCs were incubated in the presence and absence (control) of rHcARF1 $(5 \mu \mathrm{g} / \mathrm{ml})$ for 1 $\mathrm{h}$ at $37^{\circ} \mathrm{C}$. Confirmation of binding was determined by an immunofluorescence assay (IFA) as described by Yuan et al. [56]. Briefly, washed cells $\left(10^{5} / \mathrm{ml}\right)$ were fixed with $4 \%$ paraformaldehyde on a poly-L-lysine-coated glass slide. The cells were then treated with blocking solution $(4 \%$ BSA in PBS) for $30 \mathrm{~min}$ to minimize background staining. After sequential incubation with rat anti-rHcARF1 IgG $(1: 100)$ for $2 \mathrm{~h}$ and a secondary antibody (1:300) coupled to the fluorescent dye Cy3 (Beyotime, Jiangsu, China) for $1 \mathrm{~h}$, nuclear staining with 2-(4-amidinophenyl)-6-indole carbamidinedihydrochloride (DAPI, $1.5 \mu \mathrm{M}$; Sigma, MO, USA) was performed for $6 \mathrm{~min}$. Then, protein localization was determined by observing the staining patterns with a $100 \times$ oil objective lens on a laser scanning confocal microscope (L SM710, Zeiss, Jena, Germany). Digital images were captured using the Zeiss microscope software package ZEN 2012 (Zeiss, Jena, Germany).

\section{Detection of the cytokine levels by ELISA of PBMCs treated with rHcARF1}

The freshly isolated PBMCs were re-suspended to a final density of $5 \times 10^{6} / \mathrm{ml}$ in complete medium (RPMI 1640 supplemented with $100 \mathrm{U} / \mathrm{ml}$ penicillin, $100 \mu \mathrm{g} /$ $\mathrm{ml}$ streptomycin, $2 \mathrm{mM}$ L-glutamine, $10 \% \mathrm{FCS})$. In the test groups, cells were treated with ConA $(10 \mu \mathrm{g} / \mathrm{ml})$ and different concentration of the rHcARF1 (5, 10, 20, and $40 \mu \mathrm{g} / \mathrm{ml})$. The control groups were treated with ConA in equal volume of PBS or ConA and recombinant protein of empty pET32a. Then, the cells were seeded into 24well plates $\left(1 \mathrm{ml} /\right.$ well) and cultured for $24 \mathrm{~h}$ in $5 \% \mathrm{CO}_{2}$ atmosphere at $37{ }^{\circ} \mathrm{C}$. The plates were then centrifuged at $200 \times \mathrm{g}$ for $15 \mathrm{~min}$ and the supernatants were collected. The levels of IL-4, IL-10, IL-17 and IFN- $\gamma$ in supernatants were determined using commercially available goat ELISA kits (Jian cheng Biotech, China). The cell viability was assessed by means of the trypan blue exclusion test before the incubation of PBMCs with rHcARF1. Three individual experiments were performed.

\section{Cell migration assay}

The cell migration assay was performed using a Transwell system (Corning, USA), this allowed cells to migrate throughout an $8 \mu \mathrm{m}$ pore size polycarbonate membrane [27]. The treatment group was incubated with different concentrations of rHcARF1 (5, 10, 20, and $40 \mu \mathrm{g} / \mathrm{ml}$ ) and the control group was treated with an equal volume of PBS. Each experiment was performed in triplicate.

\section{Cell proliferation assay}

Cell proliferation assay was performed as previously described [64]. Briefly, $100 \mu \mathrm{l}$ of cell suspension $\left(1 \times 10^{6}\right.$ cells $/ \mathrm{ml})$ were activated with ConA $(10 \mu \mathrm{g} / \mathrm{ml})$ and a serial concentrations of $\mathrm{rHcARF} 1(5,10,20$, and $40 \mu \mathrm{g} /$ $\mathrm{ml})$. The control group was treated with ConA in equal volume of PBS. The plate was cultured at $37^{\circ} \mathrm{C}$ and $5 \%$ $\mathrm{CO}_{2}$ for $72 \mathrm{~h}$. Then $10 \mu \mathrm{l}$ of CCK-8 solutions (Beyotime Biotechnology, China) were added to each well of the plates $4 \mathrm{~h}$ before harvesting and the absorbance values at $450 \mathrm{~nm}\left(\mathrm{OD}_{450}\right)$ were measured using a microplate reader (Thermo Scientific, USA). The $\mathrm{OD}_{450}$ of controls were set as $100 \%$. Cell proliferation index was calculated by the formula: $\mathrm{OD}_{450} \mathrm{rHcARF} 1 / \mathrm{OD}_{450}$ control. Each experiment was performed in triplicate.

\section{Nitric oxide production assay}

The goat PBMCs were harvested and washed twice with PBS. Then, $100 \mu \mathrm{l}$ of cells $\left(1 \times 10^{6}\right.$ cells $\left./ \mathrm{ml}\right)$ were incubated either with PBS and a serial concentrations of rHcARF1 $(5,10,20$, and $40 \mu \mathrm{g} / \mathrm{ml})$ in 96-well plates in DMEM medium. Production of nitric oxide by PBMCs was determine by measurement of intracellular nitrite in the PBMC by using the Griess assay [65] according to the instruction of Total Nitric Oxide Assay Kit (Beyotime Biotechnology, China). Absorbance of the colored solution at $540 \mathrm{~nm}$ (OD540) in each well was measured using a plate reader (Bio-Rad Laboratories, USA). Absorbance values were converted to micromoles per liter $(\mu \mathrm{mol} / \mathrm{L})$ using a standard curve that was generated by addition of 0 to $80 \mu \mathrm{mol} / \mathrm{L}$ sodium nitrite to fresh culture media. Three individual experiments were performed.

\section{CONFLICTS OF INTEREST}

The authors declare that there is no conflict of interest.

\section{FUNDING}

The project supports were provided by the "National 
Key Basic Research Program (973 program) of China" (Grant No. 2015CB150300) and by the Priority Academic Program Development of Jiangsu Higher Education Institutions (PAPD).

\section{REFERENCES}

1. Wennerberg K, Rossman KL, Der CJ. The Ras superfamily at a glance. J Cell Sci. 2005; 118:843-46.

2. Nie Z, Randazzo PA. Arf GAPs and membrane traffic. J Cell Sci. 2006; 119:1203-11.

3. Balch WE, Kahn RA, Schwaninger R. ADP-ribosylation factor is required for vesicular trafficking between the endoplasmic reticulum and the cis-Golgi compartment. J Biol Chem. 1992; 267:13053-61.

4. Puertollano R, Randazzo PA, Presley JF, Hartnell LM, Bonifacino JS. The GGAs promote ARF-dependent recruitment of clathrin to the TGN. Cell. 2001; 105:93-102.

5. Zhou F, Dong C, Davis JE, Wu WH, Surrao K, Wu G. The mechanism and function of mitogen-activated protein kinase activation by ARF1. Cell Signal. 2015; 27:2035-44.

6. Boulay PL, Cotton M, Melançon P, Claing A. ADPribosylation factor 1 controls the activation of the phosphatidylinositol 3-kinase pathway to regulate epidermal growth factor-dependent growth and migration of breast cancer cells. J Biol Chem. 2008; 283:36425-34.

7. Schlienger S, Ramirez RA, Claing A. ARF1 regulates adhesion of MDA-MB-231 invasive breast cancer cells through formation of focal adhesions. Cell Signal. 2015; 27:403-15.

8. Zhang GF, Patton WA, Lee FJ, Liyanage M, Han JS, Rhee SG, Moss J, Vaughan M. Different ARF domains are required for the activation of cholera toxin and phospholipase D. J Biol Chem. 1995; 270:21-24.

9. Jones DH, Morris JB, Morgan CP, Kondo H, Irvine RF, Cockcroft S. Type I phosphatidylinositol 4-phosphate 5-kinase directly interacts with ADP-ribosylation factor 1 and is responsible for phosphatidylinositol 4,5-bisphosphate synthesis in the golgi compartment. J Biol Chem. 2000; 275:13962-66.

10. Cherfils J. Arf GTPases and their effectors: assembling multivalent membrane-binding platforms. Curr Opin Struct Biol. 2014; 29:67-76.

11. Boulakirba S, Macia E, Partisani M, Lacas-Gervais S, Brau F, Luton F, Franco M. Arf6 exchange factor EFA6 and endophilin directly interact at the plasma membrane to control clathrin-mediated endocytosis. Proc Natl Acad Sci USA. 2014; 111:9473-78.

12. Bruntz RC, Lindsley CW, Brown HA. Phospholipase D signaling pathways and phosphatidic acid as therapeutic targets in cancer. Pharmacol Rev. 2014; 66:1033-79.

13. Ackema KB, Hench J, Böckler S, Wang SC, Sauder U, Mergentaler H, Westermann B, Bard F, Frank S, Spang A. The small GTPase Arf1 modulates mitochondrial morphology and function. EMBO J. 2014; 33:2659-75.

14. Li Y, Kelly WG, Logsdon JM Jr, Schurko AM, Harfe BD, Hill-Harfe KL, Kahn RA. Functional genomic analysis of the ADP-ribosylation factor family of GTPases: phylogeny among diverse eukaryotes and function in C. elegans. FASEB J. 2004; 18:1834-50.

15. Serbzhinskiy DA, Clifton MC, Sankaran B, Staker BL, Edwards TE, Myler PJ. Structure of an ADP-ribosylation factor, ARF1, from Entamoeba histolytica bound to $\mathrm{Mg}(2+)-G D P$. Acta Crystallogr F Struct Biol Commun. 2015; 71:594-99.

16. Thavayogarajah T, Gangopadhyay P, Rahlfs S, Becker K, Lingelbach K, Przyborski JM, Holder AA. Alternative Protein Secretion in the Malaria Parasite Plasmodium falciparum. PLoS One. 2015; 10:e0125191.

17. Cook WJ, Smith CD, Senkovich O, Holder AA, Chattopadhyay D. Structure of Plasmodium falciparum ADP-ribosylation factor 1. Acta Crystallogr Sect F Struct Biol Cryst Commun. 2010; 66:1426-31.

18. Sahin A, Espiau B, Tetaud E, Cuvillier A, Lartigue L, Ambit A, Robinson DR, Merlin G. The leishmania ARL-1 and Golgi traffic. PLoS One. 2008; 3:e1620.

19. Peter JW, Chandrawathani P. Haemonchus contortus: parasite problem No. 1 from tropics - Polar Circle. Problems and prospects for control based on epidemiology. Trop Biomed. 2005; 22:131-37.

20. Gilleard JS. Understanding anthelmintic resistance: the need for genomics and genetics. Int J Parasitol. 2006; 36:122739.

21. Redman E, Sargison N, Whitelaw F, Jackson F, Morrison A, Bartley DJ, Gilleard JS. Introgression of ivermectin resistance genes into a susceptible Haemonchus contortus strain by multiple backcrossing. PLoS Pathog. 2012; 8:e1002534.

22. Gilleard JS. Haemonchus contortus as a paradigm and model to study anthelmintic drug resistance. Parasitology. 2013; 140:1506-22.

23. Kaminsky R, Ducray P, Jung M, Clover R, Rufener L, Bouvier J, Weber SS, Wenger A, Wieland-Berghausen S, Goebel T, Gauvry N, Pautrat F, Skripsky T, et al. A new class of anthelmintics effective against drug-resistant nematodes. Nature. 2008; 452:176-80.

24. Kotze AC. Catalase induction protects Haemonchus contortus against hydrogen peroxide in vitro. Int J Parasitol. 2003; 33:393-400.

25. Rosenfeldt F, Wilson M, Lee G, Kure C, Ou R, Braun L, de Haan J. Oxidative stress in surgery in an ageing population: pathophysiology and therapy. Exp Gerontol. 2013; 48:4554.

26. Gadahi JA, Wang S, Bo G, Ehsan M, Yan R, Song X, Xu $\mathrm{L}, \mathrm{Li}$ X. Proteomic analysis of the excretory and secretory proteins of Haemonchus contortus (HcESP) binding to goat PBMCs in vivo revealed stage-specific binding profiles. PLoS One. 2016; 11:e0159796. 
27. Price HP, Stark M, Smith DF. Trypanosoma brucei ARF1 plays a central role in endocytosis and golgi-lysosome trafficking. Mol Biol Cell. 2007; 18:864-73.

28. Volpicelli-Daley LA, Li Y, Zhang CJ, Kahn RA. Isoformselective effects of the depletion of ADP-ribosylation factors 1-5 on membrane traffic. Mol Biol Cell. 2005; 16:4495-508.

29. Price HP, Peltan A, Stark M, Smith DF. The small GTPase ARL2 is required for cytokinesis in Trypanosoma brucei. Mol Biochem Parasitol. 2010; 173:123-31.

30. Senkovich O, Chattopadhyay D. Plasmodium falciparum ARFGAP: expression and crystallization of the catalytic domain. Biochim Biophys Acta. 2004; 1698:127-30.

31. Stafford WH, Stockley RW, Ludbrook SB, Holder AA. Isolation, expression and characterization of the gene for an ADP-ribosylation factor from the human malaria parasite, Plasmodium falciparum. Eur J Biochem. 1996; 242:104-13.

32. Shi A, Grant BD. Interactions between Rab and Arf GTPases regulate endosomal phosphatidylinositol-4,5bisphosphate during endocytic recycling. Small GTPases. 2013; 4:106-09.

33. Butt AQ, Ahmed S, Maratha A, Miggin SM. 14-3-3e and 14-3-3 $\sigma$ inhibit Toll-like receptor (TLR)-mediated proinflammatory cytokine induction. J Biol Chem. 2012; 287:38665-79.

34. Ben-Addi A, Mambole-Dema A, Brender C, Martin SR, Janzen J, Kjaer S, Smerdon SJ, Ley SC. IкB kinase-induced interaction of TPL-2 kinase with 14-3-3 is essential for Toll-like receptor activation of ERK-1 and -2 MAP kinases. Proc Natl Acad Sci USA. 2014; 111:E2394-403.

35. Haque A, Noman AS, Koide N, Odkhuu E, Naiki Y, Hashimoto S, Komatsu T, Yoshida T, Yokochi T. An ADP ribosylation factor-GTPase activating protein negatively regulates the production of proinflammatory mediators in response to lipopolysaccharide. Cancer Immunol Immunother. 2011; 60:1439-46.

36. Wu JY, Kuo CC. Pivotal role of ADP-ribosylation factor 6 in Toll-like receptor 9-mediated immune signaling. J Biol Chem. 2012; 287:4323-34.

37. de Rezende MC, Araújo ES, Moreira JM, Rodrigues VF, Rodrigues JL, Pereira CA, Negrão-Corrêa D. Effect of different stages of Schistosoma mansoni infection on the parasite burden and immune response to Strongyloides venezuelensis in co-infected mice. Parasitol Res. 2015; 114:4601-16.

38. Andreasen A, Petersen HH, Kringel H, Iburg TM, Skovgaard K, Dawson H, Urban JF Jr, Thamsborg SM. Immune and inflammatory responses in pigs infected with Trichuris suis and Oesophagostomum dentatum. Vet Parasitol. 2015; 207:249-58.

39. Shakya KP, Miller JE, Horohov DW. A Th2 type of immune response is associated with increased resistance to Haemonchus contortus in naturally infected Gulf Coast Native lambs. Vet Parasitol. 2009; 163:57-66.
40. Gadahi JA, Yongqian B, Ehsan M, Zhang ZC, Wang S, Yan RF, Song XK, Xu LX, Li XR. Haemonchus contortus excretory and secretory proteins (HcESPs) suppress functions of goat PBMCs in vitro. Oncotarget. 2016; 7:35670-79. https://doi.org/10.18632/oncotarget.9589

41. Ferretti S, Bonneau O, Dubois GR, Jones CE, Trifilieff A. IL-17, produced by lymphocytes and neutrophils, is necessary for lipopolysaccharide-induced airway neutrophilia: IL-15 as a possible trigger. J Immunol. 2003; 170:2106-12.

42. da Matta Guedes PM, Gutierrez FR, Maia FL, Milanezi CM, Silva GK, Pavanelli WR, Silva JS. IL-17 produced during Trypanosoma cruzi infection plays a central role in regulating parasite-induced myocarditis. PLoS Negl Trop Dis. 2010; 4:e604.

43. Guiton R, Vasseur V, Charron S, Arias MT, Van Langendonck N, Buzoni-Gatel D, Ryffel B, Dimier-Poisson I. Interleukin 17 receptor signaling is deleterious during Toxoplasma gondii infection in susceptible BL6 mice. J Infect Dis. 2010; 202:427-35.

44. Fu Y, Wang W, Tong J, Pan Q, Long Y, Qian W, Hou $X$. Th17: a new participant in gut dysfunction in mice infected with Trichinella spiralis. Mediators Inflamm. 2009; 2009:517052.

45. Larkin BM, Smith PM, Ponichtera HE, Shainheit MG, Rutitzky LI, Stadecker MJ. Induction and regulation of pathogenic Th17 cell responses in schistosomiasis. Semin Immunopathol. 2012; 34:873-88.

46. Katawa G, Layland LE, Debrah AY, von Horn C, Batsa L, Kwarteng A, Arriens S, W Taylor D, Specht S, Hoerauf A, Adjobimey T. Hyperreactive onchocerciasis is characterized by a combination of Th17-Th2 immune responses and reduced regulatory $\mathrm{T}$ cells. PLoS Negl Trop Dis. 2015; 9:e3414.

47. Mbow M, Larkin BM, Meurs L, Wammes LJ, de Jong SE, Labuda LA, Camara M, Smits HH, Polman K, Dieye TN, Mboup S, Stadecker MJ, Yazdanbakhsh M. T-helper 17 cells are associated with pathology in human schistosomiasis. J Infect Dis. 2013; 207:186-95.

48. Rutitzky LI, Stadecker MJ. CD4 T cells producing proinflammatory interleukin-17 mediate high pathology in schistosomiasis. Mem Inst Oswaldo Cruz. 2006 (Suppl 1); 101:327-30.

49. McSorley HJ, Maizels RM. Helminth infections and host immune regulation. Clin Microbiol Rev. 2012; 25:585-608.

50. Adalid-Peralta L, Fragoso G, Fleury A, Sciutto E. Mechanisms underlying the induction of regulatory $\mathrm{T}$ cells and its relevance in the adaptive immune response in parasitic infections. Int J Biol Sci. 2011; 7:1412-26.

51. Ouyang W, Rutz S, Crellin NK, Valdez PA, Hymowitz SG. Regulation and functions of the IL-10 family of cytokines in inflammation and disease. Annu Rev Immunol. 2011; 29:71-109.

52. Xu Y, Chen W, Bian M, Wang X, Sun J, Sun H, Jia F, 
Liang C, Li X, Zhou X, Huang Y, Yu X. Molecular characterization and immune modulation properties of Clonorchis sinensis-derived RNASET2. Parasit Vectors. 2013; 6:360-360.

53. Russell JL, Powers JT, Rounbehler RJ, Rogers PM, Conti CJ, Johnson DG. ARF differentially modulates apoptosis induced by E2F1 and Myc. Mol Cell Biol. 2002; 22:1360 68.

54. Weber JD, Jeffers JR, Rehg JE, Randle DH, Lozano G, Roussel MF, Sherr CJ, Zambetti GP. p53-independent functions of the p19(ARF) tumor suppressor. Genes Dev. $2000 ; 14: 2358-65$.

55. Richter A, Löhning M, Radbruch A. Instruction for cytokine expression in $\mathrm{T}$ helper lymphocytes in relation to proliferation and cell cycle progression. J Exp Med. 1999; 190:1439-50.

56. Yuan C, Zhang H, Wang W, Li Y, Yan R, Xu L, Song X, $\mathrm{Li} \mathrm{X}$. Transmembrane protein $63 \mathrm{~A}$ is a partner protein of Haemonchus contortus galectin in the regulation of goat peripheral blood mononuclear cells. Parasit Vectors. 2015; $8: 211$.

57. Bian M, Li S, Wang X, Xu Y, Chen W, Zhou C, Chen X, He L, Xu J, Liang C, Wu Z, Huang Y, Li X, Yu X. Identification, immunolocalization, and immunological characterization of nitric oxide synthase-interacting protein from Clonorchis sinensis. Parasitol Res. 2014; 113:174957.

58. Nahrevanian H. Involvement of nitric oxide and its up/ down stream molecules in the immunity against parasitic infections. Braz J Infect Dis. 2009; 13:440-48.

59. Miljkovic D, Cvetkovic I, Vuckovic O, Stosic-Grujicic S, Mostarica Stojkovic M, Trajkovic V. The role of interleukin-17 in inducible nitric oxide synthase-mediated nitric oxide production in endothelial cells. Cell Mol Life Sci. 2003; 60:518-25.
60. Zidi S, Boulaneb-Bediar F, Belkhelfa M, Belguendouz H, Medjeber O, Henchiri C, Laouar M, Chachoua L, Lankar A, Touil-Boukoffa C. ID: 27: IL-17A and Nitric Oxide production are involved in primary Pterygium pathogenesis: A study in Algerian patients. Cytokine. 2015; 76:68-69.

61. Sun Y, Yan R, Muleke CI, Zhao G, Xu L, Li X. Recombinant Galectins of Haemonchus contortus Parasite Induces Apoptosis in the Peripheral Blood Lymphocytes of Goat. Int J Pept Res Ther. 2006; 13:387-92.

62. Han K, Xu L, Yan R, Song X, Li X. Molecular cloning, expression and characterization of enolase from adult Haemonchus contortus. Res Vet Sci. 2012; 92:259-65.

63. Wang W, Yuan C, Wang S, Song X, Xu L, Yan R, Hasson IA, Li X. Transcriptional and proteomic analysis reveal recombinant galectins of Haemonchus contortus downregulated functions of goat PBMC and modulation of several signaling cascades in vitro. J Proteomics. 2014; 98:123-37.

64. Wang W, Wang S, Zhang H, Yuan C, Yan R, Song X, Xu L, Li X. Galectin Hco-gal-m from Haemonchus contortus modulates goat monocytes and $\mathrm{T}$ cell function in different patterns. Parasit Vectors. 2014; 7:342.

65. Green LC, Wagner DA, Glogowski J, Skipper PL, Wishnok JS, Tannenbaum SR. Analysis of nitrate, nitrite, and [15N] nitrate in biological fluids. Anal Biochem. 1982; 126:13138. 